\title{
Study on Tube Profile and Heat Transfer Characteristics Under Flying-Ash Erosion
}

\author{
Yunshan Dong, Fengqi $\mathrm{Si}^{*}$ and Kun Yang \\ Key Laboratory of Energy Thermal Conversion and Control of Ministry of Education, School of Energy and Environment, Southeast \\ University, 210096 Nanjing, China
}

\begin{abstract}
Flying-ash erosion is the prime reason of the tube failure, involving the economy and safety of coal-fired power plants. To address this issue, computational fluid dynamics (CFD) is applied to this paper, which is coupled with the erosion profile calculation based on the dynamic mesh. The tube profile is analyzed under the flying-ash erosion, and the heat transfer process is further taken into account. The results indicate that as time goes on, the impact frequency and the incident angle decrease at the lower angle and increase at the higher angle. With time going on, the peak value becomes smaller at the saddle-shaped erosion rate curve, and the position of the peak value moves towards the higher angle. Meanwhile, both sides of the saddle-shaped curve expand towards the higher angle. That is, the erosion rate and the erosion loss become higher. The Nusselt number grows slightly and the trend grows faster as time goes on. The erosion profile leads to raising the temperature gradient at the angle of $0^{\circ}$, which induces the tube to rupture ultimately.
\end{abstract}

\section{Introduction}

Flying-ash erosion is the main reason of explosion in the tail heating surface of the coal-fired boiler, and it has become an industry problem that needs to be solved urgently. The tail heating surface works at the hightemperature and high-corrosive gas, which is scoured periodically by the fine-hard ash particles. This scour thins the tail heating surface and causes an uneven heat transfer for the tube, even the tube burst. It is concerned with the economy and safety of coal-fired power plants. Therefore, it is of great significance to consider the effect of the flying-ash erosion on the heat transfer, especially for the pipeline design.

Considerable studies have been performed on erosion. Finnie et al [1-4] presented the work focusing on theoretical erosion mechanism. Based on the cutting theory, they classified the erosion as cutting and deformation to predict the erosion rate as a function of particle velocity, incident angle and the tensile properties of the target material and so forth. Bauver et al. [5] studied the erosion rate of fly ash particles on the surface of the single tube through the single tube test. It was found that the maximum wear rate occurred at the angle of $36 \sim 40^{\circ}$. Based on the experimental results by Bauver, Lee et al. [6]determined the relevant correlation of erosion rate and calculated the erosion rate of the tube by discrete phase model and discrete element method. Zhao et al.[7-9] studied the influence of ash particle size, temperature and velocity on the erosion characteristics in power plant pipes. These studies are based on the jet flow test, and obtained the correlation for flying-ash erosion in the power plant. Moreover, in terms of heat transfer, Žukauskas et al. [10] summarized the heat transfer correlations, which are appropriate to the single tube through a series of experimental results. Khan et al. [11] solved the momentum equation and gave an approximate solution for the heat transfer around the tube, which referred to the Von Karman-Pohlhausen method.

The aforementioned studies have independently studied the erosion and the heat transfer characteristics of the tube, not completely combining two parts. That is, the erosion profile is difficult to obtain. Thus, CFD (computational fluid dynamics) is applied to this paper, which integrates the erosion profile based on the dynamic mesh. The tube profile is analyzed at different time, and the evolution process of heat transfer is future taken into account. Moreover, this study will benefit the design of the economizer and guide the operation.

\section{Model descriptions}

\subsection{Physical Model}

A single tube is studied in this paper, whose structural parameters refer to the experiment conducted by Ref.[5]. The tube adapts to the economizer of the coal-fired boiler, improving the overall thermal efficiency as the gas-water exchangers, exacting residual heat energy

\footnotetext{
* Corresponding author: fqsi@seu.edu.cn
} 
from the flue gas and transferring the energy to the feed water. The computational physical domain is shown in Figure 1, where $D$ is the external diameter of the single tube. In order to eliminate the effect of the external boundary on the single pipe, the inlet region and the outlet region are respectively extended. The other walls are ensured to be far away from the region of the single tube. Table 1 gives the relevant parameters of the physical domain. The physical parameters correspond to $100 \%$ of the boiler load under turbine heat acceptance. Besides, the particle size refers to the experimental size in Ref.[5].

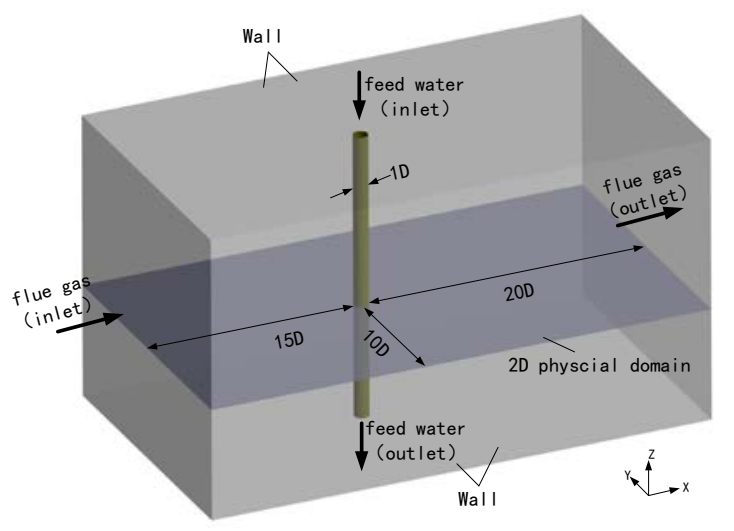

Fig. 1. Computational physical domain.

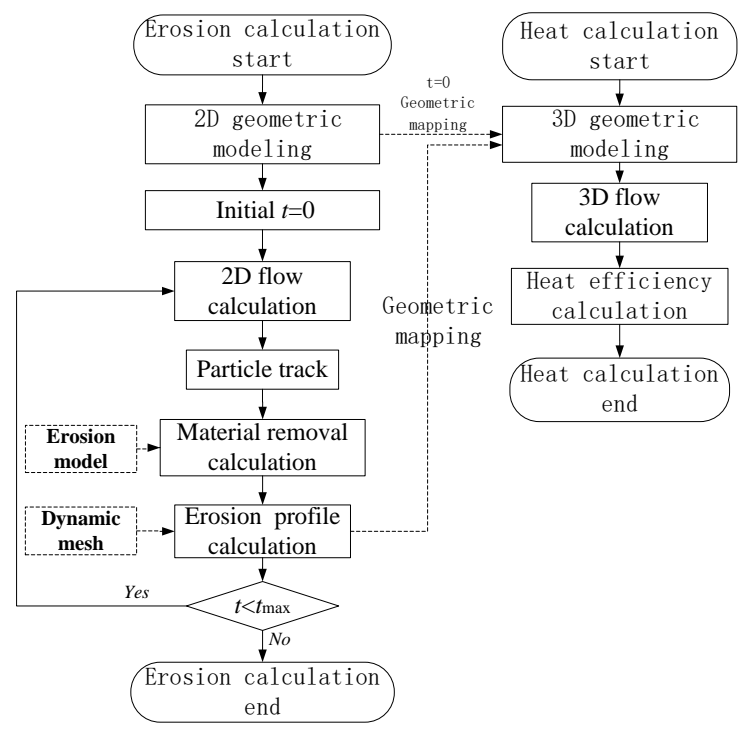

Fig. 2. Calculative process of single tube.

The calculative process is divided into the erosion profile calculation and the heat transfer characteristics calculation in Figure 2. This calculation of erosion profile refers to Ref.[12]. In Ref.[12], the unsteady calculation is adopted, and it is considered that the erosion effect of the time step amounts to one day. Erosion only occurs on the interface between the gassolid domains. Therefore, the calculation of erosion profile can neglect the influence of the tube and the feed water, and only the physical domain of the flue gas is retained and calculated. The erosion model is added to the calculative process by the user defined function (UDF), and the erosion profile is calculated by the dynamic mesh technology. The heat transfer calculation is a one-way coupling to map the two-dimensional erosion profile to a three-dimensional model at different time, as shown in Figure 1. Meanwhile the feed water and solid domains are added to the heat transfer characteristics calculation. At last the heat transfer characteristic is calculated by CFD. The mapping is not a two-way coupling and is a micro gap against the reality. However the laws from the one-way coupling is consistent with the two-way coupling.

\subsection{Grid Discretization and Numerical solution}

\subsubsection{Grid discretization}

Erosion only occurs at the interface, and the influence of the feed water can be neglected. Therefore, the erosion profile calculation only discretizes the gas domain. In order to accurately capture the information of the flow field, the structured mesh is used to discretize the physical domain. Considering the effect of the boundary layer on the calculation, the grid of the tube wall is refined and the far field adopts the sparse grid in Figure 3. This grid can save the computing time and ensure the accuracy.

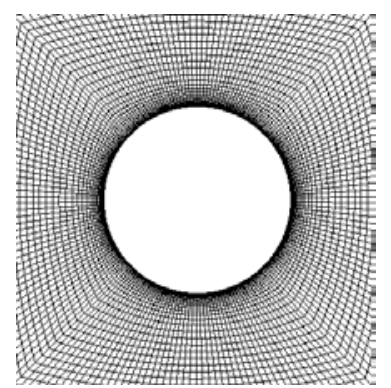

Fig. 3. Refined grid near wall.

In the heat transfer characteristics calculation, the profile at different time is introduced from the erosion profile calculation. Besides, the flue gas, the tube and the feed water domains are meshed. The information is exchanged through the interface.

\subsubsection{Governing equation}

The erosion profile calculation includes fluid motion, particle motion, particle collision, particle wear model, and the heat transfer characteristics calculation has the models of the fluid motion and the thermal conductivity. Among them, the particle collision model and the erosion model are as follows.

\section{1)particle collision model}


The particle collision includes the particle-particle collision and the particle-wall collision. Due to the particle concentration in tail heating surface is relatively low, the particle-particle collision can be neglected. Only particle-wall collision is remained, as shown in Figure 4.

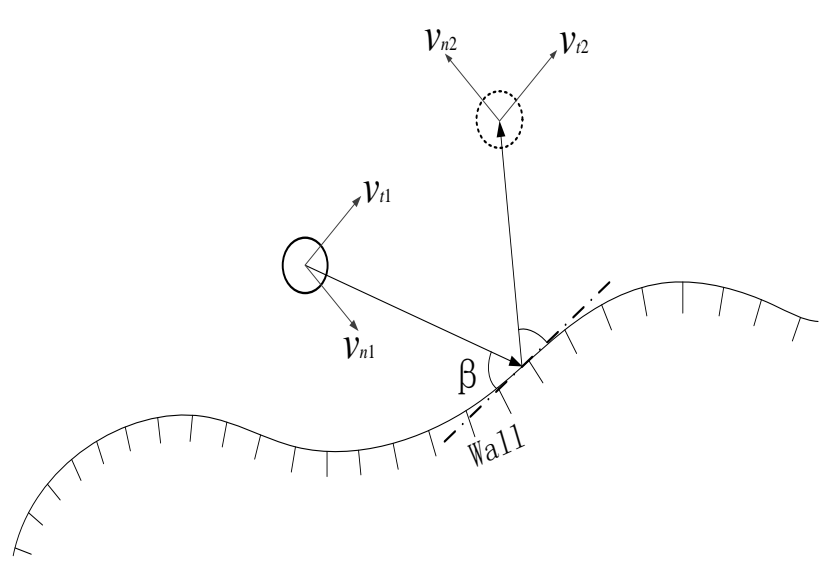

Fig. 4. Schematic of particle-wall collision.

The general particle collision model given by Ref.[13] is as follows:

$$
\begin{aligned}
& v_{n 2} / v_{n 1}=0.993-1.76 \beta+1.56 \beta^{2}-0.49 \beta^{3} \\
& v_{t 2} / v_{t 1}=0.998-1.66 \beta+2.11 \beta^{2}-0.76 \beta^{3}
\end{aligned}
$$

where, $v_{n i}$ and $v_{t i}$ are the normal and tangential velocity of the particle, subscript $i=1$ represents the velocity before collision, $i=2$ represents the velocity after collision, $\beta$ is the incident angle of the particle.

\section{2) Erosion model}

The erosion model is added by the UDF of Fluent. According to the experimental data by Ref.[5], the erosion model is constructed by, as follows.

$$
E=K C_{p} f(\beta) V_{1}^{n} g\left(d_{p} / 200\right)
$$

where,

$$
\begin{aligned}
& f(\beta)=a+b \beta+c \beta^{2}+d \beta^{3}+e \beta^{4}+f \beta^{5} \\
& g(x)=g\left(d_{p} / 200\right)=1-e^{-17.387(x-0.025)}
\end{aligned}
$$

where, $E$ is the erosion rate, $K$ is the erosion constant, $V_{1}$ is the velocity magnitude before collision, $n$ is the velocity constant. $C_{p}$ is the particle concentration, $f(\beta)$ is a function of the incident angle, the constants in Eq.(4) refer to the constants by Ref.[6]. $d_{p}$ is the particle diameter, $g\left(d_{p} / 200\right)$ is a function the particle diameter.

Figure 5 gives the relation between $f(\beta)$ and $\beta$. SS 304 is a ductile metal, which corresponds to the law of the erosion rate for the ductile metal. Namely, as $\beta$ increases, $f(\beta)$ increases first and then decreases. Besides, for the material of SS 304, the maximum appears at the angle of $30^{\circ}$.

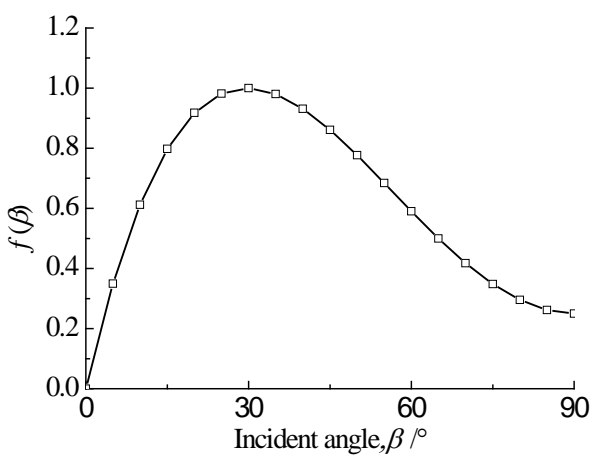

Fig. 5. Relation between $f(\beta)$ and $\beta$.

\section{3)Solver settings}

The gas is regarded as compressible turbulent flow. The feed water has no phase transition, which is regarded as uncompressible flow. Besides, the boundaries are set as the velocity inlet, the pressure outlet. The corresponding parameters are given in Table 1. The turbulence model adopts the standard $k-\varepsilon$ model. In the erosion profile calculation, the particle size ranges from $10 \mu \mathrm{m}$ to $150 \mu \mathrm{m}$, which satisfies the RosinRammler distribution. In the heat transfer characteristics calculation, the interface between the fluid domain and the solid domain satisfies the following conditions:

$$
\left\{\begin{array}{c}
T_{f, b}=T_{s, b} \\
Q_{f, b}=-Q_{s, b}
\end{array}\right.
$$

where, $T_{f, b}$ and $T_{s, b}$ are respectively the temperature of the fluid and solid interfaces, $Q_{f, b}$ and $Q_{s, b}$ are respectively the heat flow of the fluid and solid interfaces.

At the same time, the distributed solver is used to solve the governing equation. When the calculative residual reaches $10^{-5}$ and the fluctuation of the corresponding parameter is less than $3 \%$ in the flow field, it is likely that the calculation is convergent.

\section{Validations}

In order to verify the accuracy of the model, this paper compares the calculated results of the erosion profile and the heat transfer characteristics, as shown in Figure 6 and Figure 7. For Reynolds number $R e$ and Nusselt number $\mathrm{Nu}$, the qualitative temperature is $\left(t_{w}+t_{\infty}\right) / 2 . t_{w}$ is the average temperature of the outer wall on the tube, and $t_{\infty}$ is the temperature of the free stream. The characteristic length $d$ is the external diameter of the single tube. The characteristic velocity is the velocity of the free stream. $\theta$ 
is the collision position, as shown in Figure 6. The validation indicates that the error between the numerical results and the experiment $[10,14,15]$ is within the allowable range, both for the saddle-shaped erosion rate and for the heat transfer. Thus, this model satisfies the computing requirements.

Table 1. Physical parameters

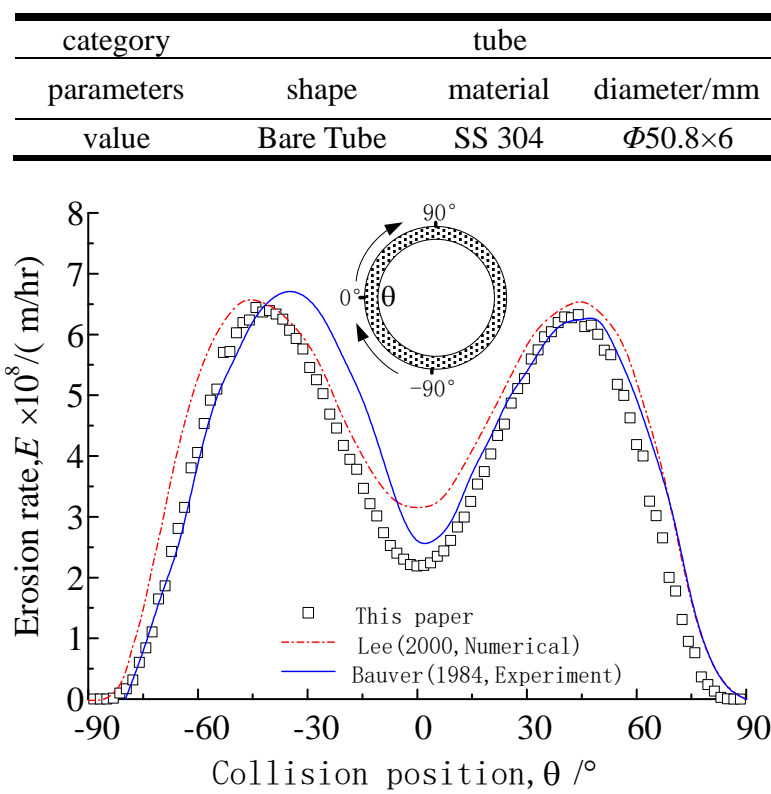

Fig. 6. Validation of erosion characteristics.

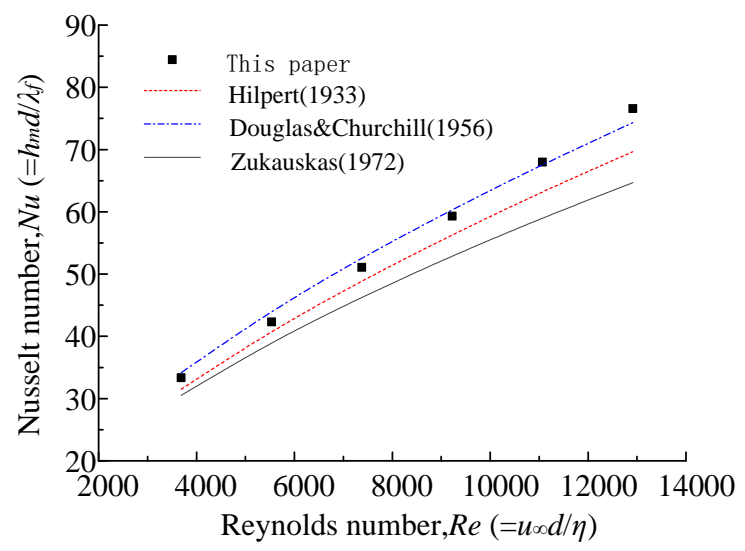

Fig. 7. Validation of heat transfer characteristics.

\section{Results and Discussion}

\subsection{Particle motion characteristics}

Figure 8 shows the normalized collision frequency for ash particle. The criterion is the maximum collision frequency of the origin tube at the collision position of $\theta=0^{\circ}$. The collision frequency presents an arch shape. Namely, as the collision position increases, the collision frequency decreases. However, compared with the small size particle, the collision frequency of the large particle is relatively flat around the low angle of the collision position. This is mainly because the inertia of the large particle is larger, resulting in that the gas flow is difficult to change the motion direction of the particle. Therefore the particle is uniformly distributed to impact the tube.

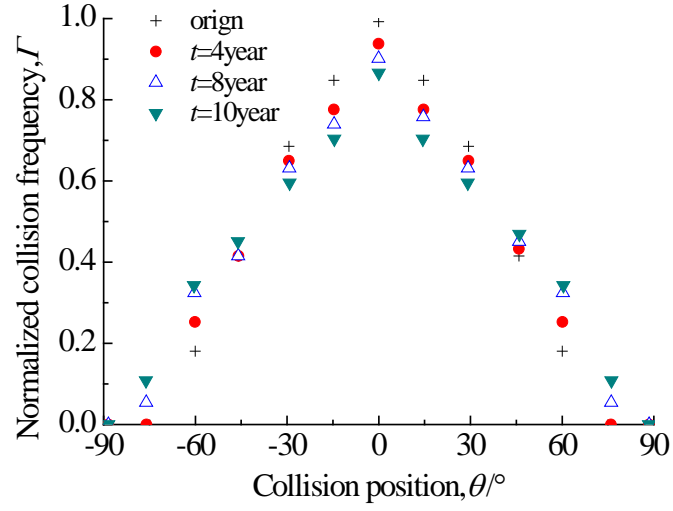

(a)

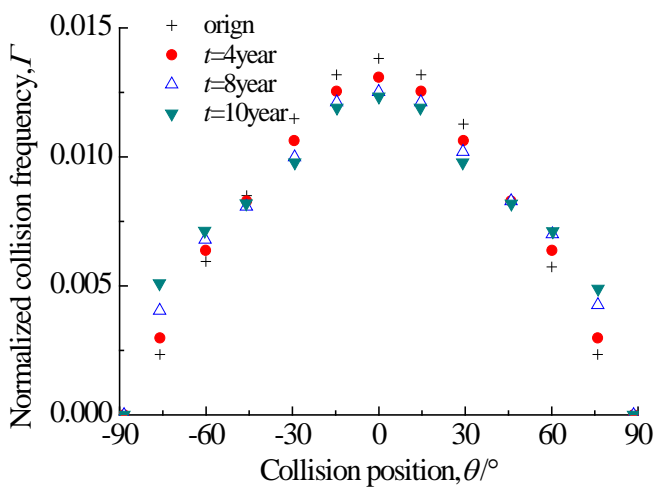

(b)

Fig. 8. Normalized collision frequency for ash particle: (a) $d_{p}=50 \mu \mathrm{m}$, (b) $d_{p}=130 \mu \mathrm{m}$.

Figure 9 shows the average incident angle of the ash particles at different collision locations. The trend of the incident angle is consistent with the collision frequency for the tube. As time goes on, the flow velocity near the 
wall is increased at the small angle of collision position, and the ability of the particles to cross the boundary layer is reduced. It results in the incident angle decreasing. For the large angle of collision position, due to the erosion profile, the incident angle is shifted, and the incident angle becomes increasing.

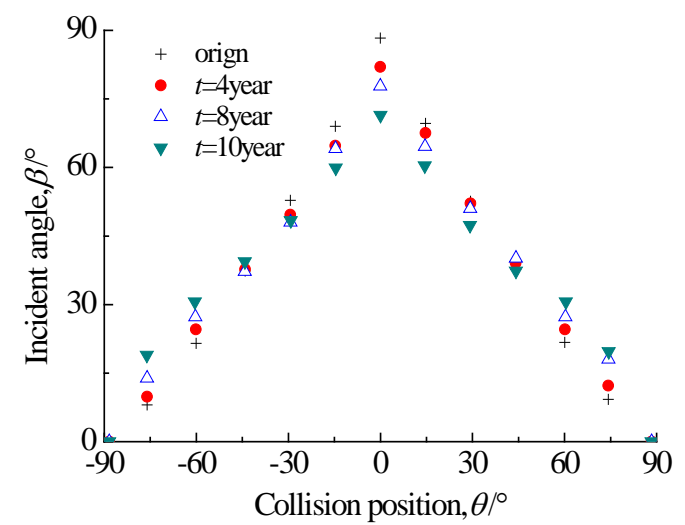

Fig. 9. Incident angle of the ash particles.

\subsection{Erosion profile}

Figure 10 shows the erosion profile for the tube. The erosion profile calculation can accurately simulate the evolution of the erosion profile in this paper. In addition, the erosion region mainly occurs on the windward side of the tube. As the angle of the collision position increasing, the erosion depth increases first and then decreases. The maximum appears at $35^{\circ} \sim 40^{\circ}$. It attributes to the maximum of the single particle at the angle of $30^{\circ}$.

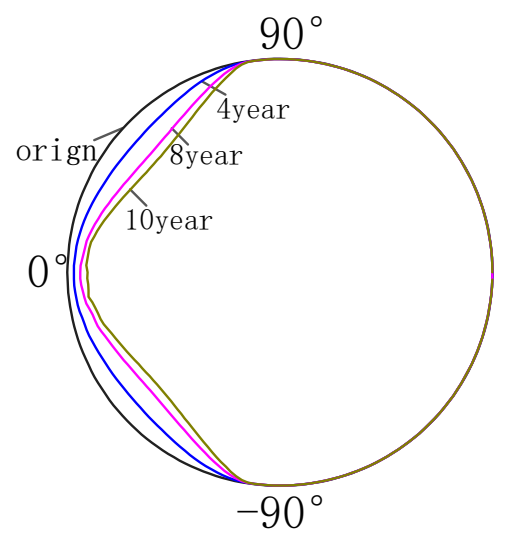

Fig. 10. Erosion profile for the tube.

At the same time, as time goes on, the erosion depth increases. The shape of the windward side gradually turns from a semicircle to a V-shape. This shape not only favors the flow of gas, but also allows more particles to move to a large angle of collision position.

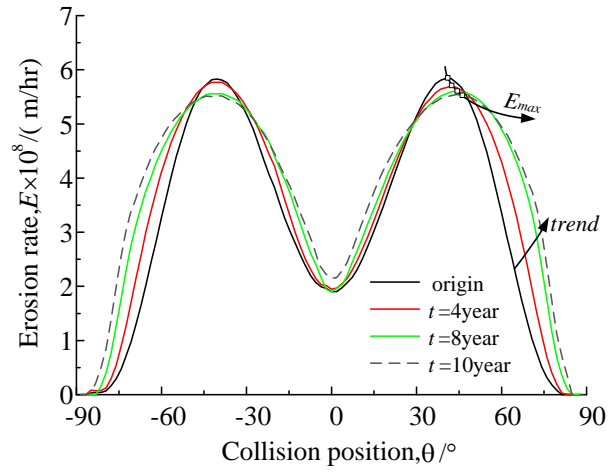

Fig. 11. Evolution of erosion rate for single tube.

Figure 11 shows the evolution of the erosion rate for the single tube. As time goes on, the peak value becomes smaller at the saddle-shaped curve of erosion rate, and the position of peak value moves towards the higher angle. It is concerned that the erosion profile is more favourable to the particle flow. Numerous particles turn to the high angle of collision position, and the probability of the particles colliding increases at the high angle. Thus, both sides of the saddle-shaped curve expand towards the higher angle. However the middle of the saddle-shaped curve expands towards the lower angle. It is noted that the variation of erosion rate is not obvious at the small angle. As time goes on, the particle collision frequency decreases at a small angle, but the incident angle correspondingly decreases. According to Figure 5, with the incident angle increased, the erosion rate increases first and then decreases. At the small angle of collision position, the incident angle goes down, and the corresponding erosion rate of the single particle is increasing. In general, the erosion rate is determined by the relative change of the particle collision frequency and the incident angle, which leads to the curve movement.

In addition, the area integrated by the erosion rate represents the total amount of erosion loss for the tube. As time goes on, the total amount of erosion loss goes up. It is harmful to the tube protection.

\subsection{Heat transfer characteristics}

Figure 12 shows the variation of the Nusselt number for the single tube. As time goes on, the Nusselt number shows a slight upward trend, and gradually becomes faster. The profile is conducive to the fluid flow, which leads the flow velocity near the wall to increase. The high velocity flow increases the heat transfer. Meanwhile, the amount of erosion loss increases, equivalent to the rapid decline for the average thickness of the tube. The thinning trend of the tube leads to a rapid growth for the Nusselt number. However, it is worth noting that since the thermal resistance of the tube is small relative to the flue gas and the feed water, the variation of the Nusselt number is relatively slow. 


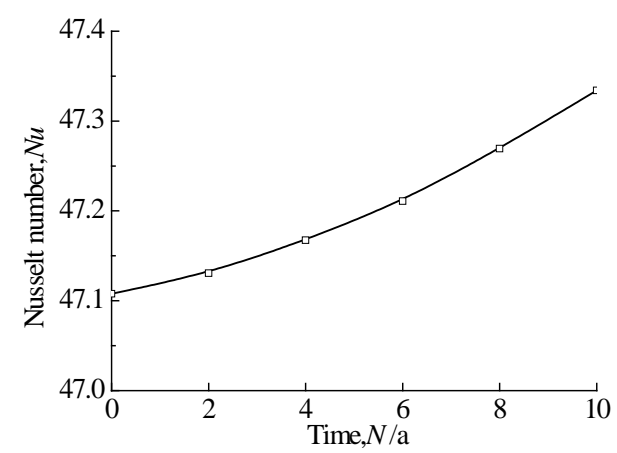

Fig. 12. Variation of Nusselt number for single tube.

The thermal stress and the compressive stress of the feed water on the tube are the prime reason of tube failure for the heating surface. Figure 13 shows the stress condition of the tube, where $\sigma_{\theta}$ and $\sigma_{r}$ are respectively the tangential and radial force at a certain position. The corresponding expressions are as follows:

$$
\begin{gathered}
\sigma_{\theta}=\frac{P d}{2 \delta}+\sigma_{\theta, \text { heat }} \\
\sigma_{r}=\frac{P}{2}+\sigma_{r, \text { heat }}
\end{gathered}
$$

where, $P$ is the pressure of the feed water, $d$ is the internal diameter of the tube, $\delta$ is the tube thickness, $\sigma_{\theta \text {, heat }}$ is the tangential component of the thermal stress, $\sigma_{\theta \text {, heat }}$ is the radial component of the thermal stress .

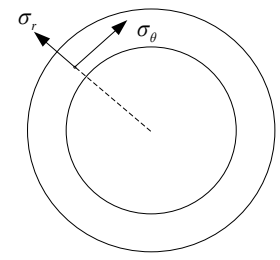

Fig. 13. Stress condition of single tube.

According to the theory of the maximum shear stress, the strength conditions of the elastic failure criterion are

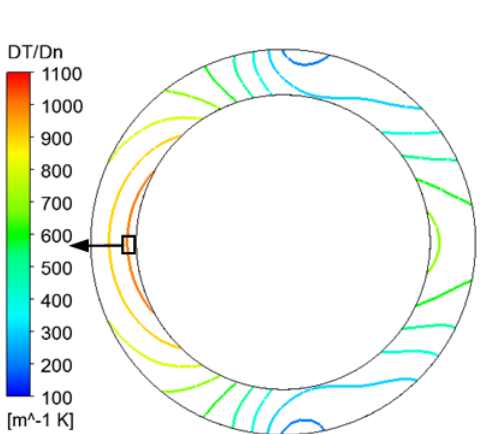

(1) origin
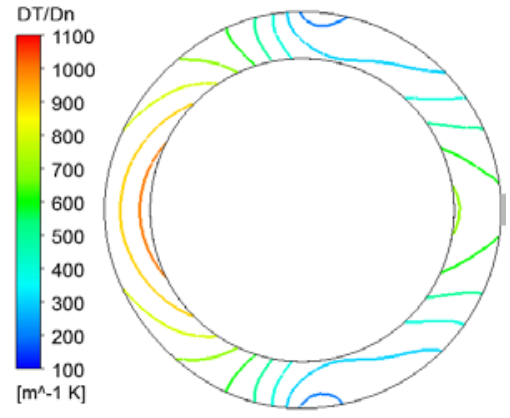

(2) $t=2$ year $\sigma_{\theta}-\sigma_{r} \leq[\sigma],[\sigma]$ is the allowable stress. If the Eqs.(7)-(8) do not take into account the effects of thermal stress, the most dangerous region is the thinnest position of the tube. Namely, the position of tube bursting is the severest erosion region $\left(\theta=35^{\circ} \sim 40^{\circ}\right)$. However the burst position in field is at the center of the front $\left(\theta=0^{\circ}\right)$, as shown in Figure 14. The cause of the difference is the neglect of the effect of thermal stress on the tube.

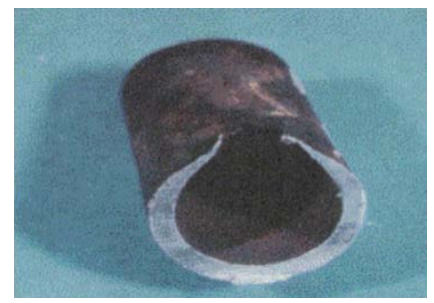

Fig. 14. Burst position of economizer in the field.

Figure 15 shows the temperature gradient distribution for the tube. The temperature gradient causes the uncoordinated thermal deformation of the tube, which forms thermal stress. The maximum of the temperature gradient appears at the low angle of collision position $\left(\theta=0^{\circ}\right)$. However compared to the low angle of collision position, the temperature gradient is much smaller for the most serious erosion region, whose angle of collision position is between $35^{\circ}$ and $40^{\circ}$. That is, the thermal stress is the largest at the low angle of collision position, and the thermal stress in the severest worn region is not very large. In addition, compared to the region 1 and the region 2 in Figure 14(1) (6), the temperature gradient gradually increases at the low angle of collision position as time goes on, but the temperature gradient is a downward trend at the severe erosion region. It shows that flying-ash erosion not only thins the front thickness at the low angle of collision position, but also increases the stress of the feed water on the tube; Flying-ash erosion thins the front thickness at the severe erosion region, but increases the thermal stress. Combined with the burst position, it can be considered that the thermal stress is the decisive factor for the tube rupture by flyingash erosion. 

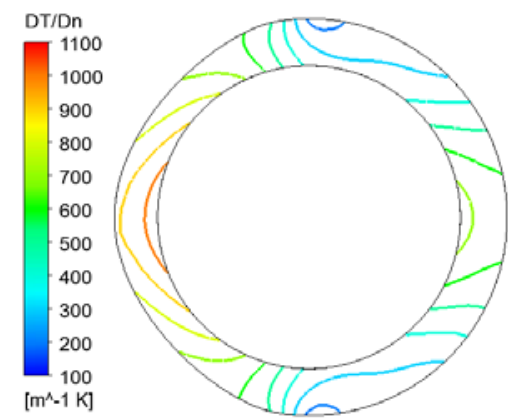

(4) $t=6$ year

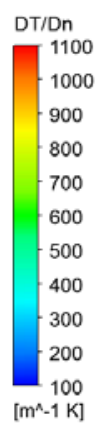

$\left[\mathrm{m}^{\wedge}-1 \mathrm{~K}\right]$

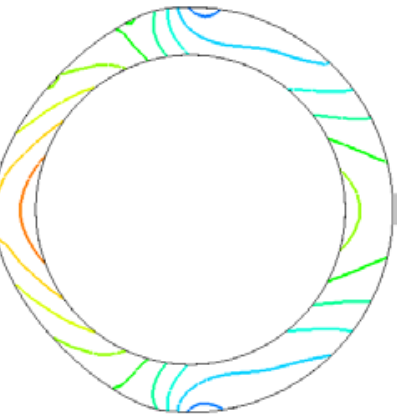

(5) $t=8$ year

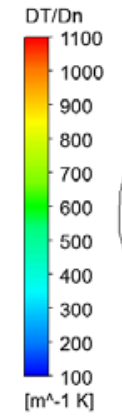

$\left[\mathrm{m}^{\mathrm{n}}-1 \mathrm{~K}\right]$

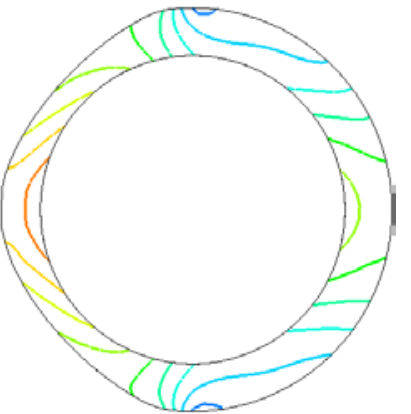

(6) $t=10$ year

Fig. 15. Temperature gradient distribution for single tube.

In this paper, the temperature gradient line displacement is applied to estimating the trend of the maximum temperature gradient at the angle of collision position $\left(\theta=0^{\circ}\right)$. The temperature gradient line moves to the outside of the pipe wall, Due to the maximum temperature gradient induces the tube to rupture, it means that the position of $\theta=0^{\circ}$ is more dangerous. Thus it is preferable to use the displacement of the temperature gradient. Figure 16 shows the displacement of the temperature gradient line, of which value is $1000 \mathrm{~K} / \mathrm{m}$. As time goes on, the displacement rate of the temperature gradient line decreases, and the isothermal gradient line tends to be stable.

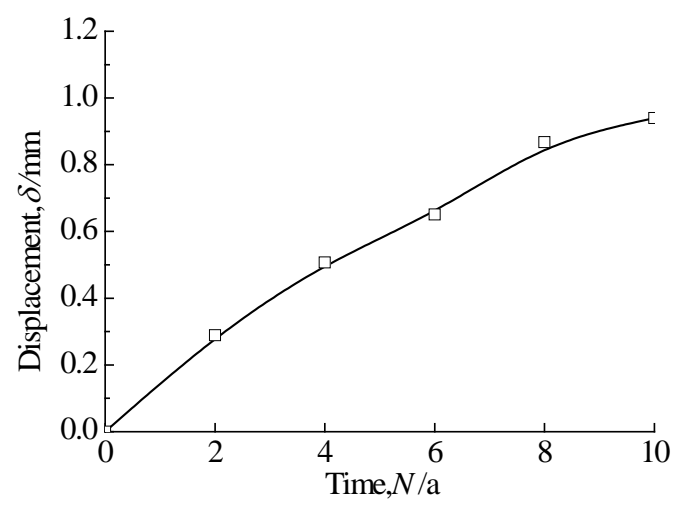

Fig. 16. Displacement of temperature gradient line, ( $D T / D n$ $=1000 \mathrm{~K} / \mathrm{m}$ ).

\section{Conclusions}

This paper gives the tube erosion profile and heat transfer characteristics. The tube erosion profile is obtained through the dynamic mesh. The heat transfer characteristics are a one-way coupling to map the twodimensional erosion profile to a three-dimensional model at different time. As a result, the impact frequency and the incident angle decrease at the lower angle and increase at the higher angle as time goes on. Similarly, the peak value becomes smaller at the saddle-shaped erosion rate curve, and the position of peak value moves towards the higher angle. Meanwhile, both sides of the saddle-shaped curve expand towards the higher angle, namely the higher erosion rate at the higher angle, or that the erosion loss becomes more serious. The Nusselt number grows slightly and its trend grows faster as time goes on. The erosion profile leads to raise the temperature gradient at the angle of $0^{\circ}$, which induces the tube to rupture ultimately. Nevertheless, for the most serious erosion region at the angle of $35^{\circ} \sim 40^{\circ}$, the effect of erosion on thermal stress decreases.

\section{References}

1. I. Finnie, Wear 3, 87 (1960)

2. J. G. A. Bitter, Wear 6, 5 (1963)

3. C. Huang, S. Chiovelli, P. Minev, J. Luo, and K. Nandakumar, Powder Technol. 187, 273 (2008)

4. Y. Ben-Ami, A. Uzi, and A. Levy, Powder Technol. 301, 1032 (2016)

5. W. Bauver, J. Bianca, J. Fishburn, and J. Mcgowan, in 1984 Jt. Power Gener. Conf. GT Pap. (1984), pp. 84-JPGC-FU-3

6. B. E. Lee, C. A. J. Fletcher, and M. Behnia, Wear 240, 95 (2000)

7. X. Zhao, G. Ye, C. Zhu, and J. Sun, J. Shanghai Univ. Electr. Power 32, 1 (2016)

8. X. Zhao and J. Sun, Proc. CSEE 25, 117 (2005)

9. X. Zhao, G. Ye, and J. Sun, J. Shanghai Univ. Electr. Power 34, 101 (2018)

10. A. Žukauska, Advances in Heat Transfer (Academic Press, New York, 1972)

11. W. A. Khan, J. R. Culham, and M. M. Yovanovich, J. Heat Transfer 127, 785 (2005)

12. Y. Dong, Z. Qiao, F. Si, B. Zhang, C. Yu, and X. Jiang, Catalysts 8, (2018)

13. G. Grant and W. Tabakoff, J. Aircr. 12, 471 (1975)

14. R. Hilpert, Forsch Geb Ingenieurwes 4, 215 (1933)

15. M. J. M. Douglas and S. W. Churchill, Chem. Eng. Prog. 52, 23 (1956) 